\title{
Research on Depression of Postgraduates in Universities Take Zhejiang For Example
}

\author{
Huang Tao, Gu Rong, Wang Yulong \\ Zhejiang University of Technology, Hangzhou, Zhejiang, 310023, China (huangtao21s@ @otmail.com)
}

\begin{abstract}
With heavy research and study pressure, the postgraduate is likely to get depression. This paper presents the survey result of 500 postgraduates who studied in Zhejiang province based on BDI-II(Beck Depression Inventory). Survey result shows the depression ratio of Zhejiang is $\mathbf{1 5 . 6 \%}$. Further comparisons and analyses are made in the four items: gender, grade, major, source of students. Based on the inventory, some suggestions are given for universities' mental health work.
\end{abstract}

Keyword-depression, mental health, postgraduate, BDI-II

\section{高校硕士研究生抑郁现状分析及对策研究 \\ 一以浙江省为例}

\author{
黄 涛 顾 容 王玉龙 \\ 浙江工业大学教育科学与技术学院, 杭州, 浙江, 中国
}

\begin{abstract}
摘 要 研究生科研任务繁重, 学习压力大, 容易导致抑郁。本研究以浙江省为例, 运用Beck抑郁问卷(第二版)对浙江省10所高 校, 共500名硕士研究生进行了问卷调查, 抑郁检出率为 $15.6 \%$ 。研究从性别、年级、专业、生源这四个条目对比了抑郁状况的差异, 并进行相关分析讨论。基于调研结果, 对高校的研究生心理健康工作提出相应对策。
\end{abstract}

关键词 抑有, 心理健康, 研究生, 贝克抑郁量表第二版

1. 引言

抑郁是一种心境异常低落、不愉快的负性情绪状态。 抑郁会给人们的日常生活和学习工作带来消极影响, 若长 期处于抑郁状态, 则会导致心理障碍的发生。研究指出, 抑郁在正常人群中的发生率呈不断上升趋势, 已成为 21 世 纪影响人类身心健康的主要因素之一 [1]。研究生科研繁重, 学习压力大, 极易出现各种心理健康问题, 这给高校的研 究生心理健康教育工作带来巨大挑战。有关研究生心理问 题的研究结果显示, 在检出率较高的前 4 种问题中均包含 抑郁[2], 表明抑郁是影响研究生心理健康的主要因素。随 着高校全日制硕士研究生招生规模的不断扩大, 有必要对 其心理状况进行了解, 为进一步做好研究生心理健康教育 工作提供依据。本研究采用 Beck 抑郁量表, 以浙江高校全 日制硕士研究生为研究对象, 对其抑有状况进行调研分析, 根据调研结果为高校的研究生健康教育提出对策。

\section{2. 研究对象与方法}

\section{1 研究对象}

本研究以浙江高校在读全日制硕士研究生为研究对 象, 采用 Beck 抑有量表第二版通过纸质问卷和网上问卷的 形式随机选取来自浙江大学、浙江工业大学、浙江理工大 学、浙江工商大学、宁波大学、浙江林业大学、浙江师范 大学、杭州师范大学、杭州电子科技大学和浙江传媒学院 等 10 所高校的 500 名硕士研究生发放问卷。共回收问卷 487 份, 回收率为 $97.4 \%$, 其中有效问卷 456 份, 有效率为 93. 6\%。根据性别、年级、专业和生源对样本进行统计分类, 研究对象的一般情况见表 1 。 
表 1 样本基本情况

\begin{tabular}{|c|c|c|c|}
\hline \multicolumn{2}{|c|}{ 变量 } & 人数 & 百分比 $(\%)$ \\
\hline \multirow{2}{*}{ 性别 } & 男 & 190 & 41.7 \\
\cline { 2 - 4 } & 女 & 266 & 58.3 \\
\hline \multirow{3}{*}{ 年级 } & 研一 & 255 & 55.9 \\
\cline { 2 - 4 } & 研二 & 145 & 31.8 \\
\cline { 2 - 4 } & 研三 & 56 & 12.3 \\
\hline \multirow{3}{*}{ 专业 } & 理工科 & 303 & 66.4 \\
\cline { 2 - 4 } & 文科 & 153 & 33.6 \\
\hline \multirow{3}{*}{ 生源 } & 农村 & 267 & 58.5 \\
\cline { 2 - 4 } & 县镇 & 107 & 23.5 \\
\cline { 2 - 4 } & 城市 & 82 & 18.0 \\
\hline
\end{tabular}

\section{2 研究工具}

采用 Beck 抑郁量表第二版(BDI-II)中文版对上述对象 进行调查。贝克抑郁量表第二版是 1996 年贝克根据 DSM-IV 抑郁症诊断标准在第一版基础上修订而成[3]。该 量表共包含 21 项条目, 各条目均有 4 个短句, 分别为 0-3 分四级评分。要求被调查者在每项条目中选择最符合自己 心情/情况(最近一周, 包括今天)的一项并记分, 最后累加 得出总分。按量表总得分来区分抑郁程度: 0-13 分为无抑 郁, 14-19 分为轻度抑郁, 20-28 分为中度抑郁, 29-63 分 为重度抑郁, 分数越高, 抑郁程度越严重。BDI-II 中文版 的内部一致性系数为 0.94 , 重测相关系数为 0.55 , 与汉密 尔顿抑郁量表相关系数达到 0.67 , 表明贝克抑郁量表第二 版中文版具有良好的信度和效度[4]。

\section{3 实施过程及数据处理}

纸质问卷由心理调查员发放, 网络问卷通过问卷星在 线问卷调查平台发放。为保证本研究的信度和效度, 聘请 的心理调查员均为研究生心理协会会员或心理学专业学 生, 调查前对心理调查员进行相关指导。所得数据统一在 SPSS18 上进行统计处理。

\section{3. 研究结果}

\section{1 研究生抑郁总体状况}

统计结果显示, 量表平均得分为 7.55 , 标准差为 7.535 。 所抽取样本的各抑郁等级人数分布情况为: 无抑郁人数为 385 人, 占 $84.4 \%$; 轻度抑郁 39 人, 占 $8.6 \%$; 中度抑郁 22 人, 占 $4.8 \%$; 重度抑郁 10 人, 占 $2.2 \%$ (见表 2 )。
表2 Beck抑郁量表得分情况

\begin{tabular}{|c|c|c|c|c|c|}
\hline 类别 & 抑郁程度 & 人数 & 百分比 & 均值 & 标准差 \\
\hline $0-13$ & 无抑有 & 385 & $84.4 \%$ & 4.99 & 4.031 \\
\hline $14-19$ & 轻度抑郁 & 39 & $8.6 \%$ & 16.64 & 1.724 \\
\hline $20-28$ & 中度抑郁 & 22 & $4.8 \%$ & 24.05 & 2.081 \\
\hline $29-63$ & 重度抑郁 & 10 & $2.2 \%$ & 34.40 & 7.058 \\
\hline
\end{tabular}

表 3 呈现了Beck抑郁量表各项条目中得分大于 2 分的人 数、得分均值及标准差。条目得分大于 2 分表明调查对象在 该项目所对应的状态中存在较为明显的问题。从表中我们 可以看到得分大于 2 , 人数最多的项目是自责, 其次是睡眠 改变, 最少的是自杀意向。在各条目中, 得分均值最高的 是睡眠改变, 其次是疲乏, 这表明大部分研究生存在睡眠 和身体疲乏的问题。

表3 Beck抑郁量表各条目得分情况

\begin{tabular}{|l|c|c|l|c|c|}
\hline \multicolumn{1}{|c|}{ 条目 } & 得分 $\geq 2$ & 均值 & \multicolumn{1}{|c|}{ 条目 } & 得分 $\geq 2$ & 均值 \\
\hline 1.悲伤感 & 11 & .24 & 2.悲观 & 41 & .30 \\
\hline 3.失败感 & 29 & .28 & 4.无愉快感 & 28 & .29 \\
\hline 5.内疚感 & 16 & .45 & 6.惩罚感 & 36 & .42 \\
\hline 7.自我嫌弃感 & 49 & .29 & 8.自责 & 71 & .52 \\
\hline 9.自杀意向 & 4 & .12 & 10.易哭泣 & 39 & .32 \\
\hline 11.激越 & 17 & .38 & 12.兴趣缺乏 & 22 & .46 \\
\hline 13.犹豫不决 & 24 & .36 & 14.无价值感 & 24 & .21 \\
\hline 15.精力不足 & 24 & .52 & 16.睡眠改变 & 53 & .65 \\
\hline 17.易发火 & 10 & .26 & 18.食欲改变 & 17 & .34 \\
\hline 19.注意困难 & 23 & .43 & 20.疲乏 & 29 & .53 \\
\hline 21.性欲缺乏 & 21 & .19 & \multicolumn{3}{|l}{} \\
\hline
\end{tabular}

3.2 研究生抑郁状况的人口学变量分析

本次调研从性别、年级、专业和生源地这 4 因素对数 据进行分组讨论, 对比各组抑郁检出率及在不同条目上的 得分差异。

\section{（1）不同性别研究生抑郁状况}

图 1 呈现了不同性别研究生的抑郁状况差异。据图可 知, 男生的抑郁百分比为 $18.9 \%$, 大于女生 $13.2 \%$ 抑郁率。 值得注意的是, 男生中度和重度抑郁比例均高于女生, 表 明男生抑郁状况较女生更为严重。以性别为分组变量, 通 过独立样本 $\mathrm{T}$ 检验, 对男女生抑郁状况进一步分析。表 4 给出了存有显著差异的项目及男女总分, 其余项目由于男 女生得分不存在显著差异, 在表中不予列出。检验发现男 
女生在失败感、内疚感、惩罚感和易哭泣这四个项目上得 分存在显著差异。其中在惩罚感得分上差异非常显著, 在 其余三项上得分差异显著。男生量表平均得分为 8.22 高于 女生平均分 7.08 , 两者统计差异不显著。

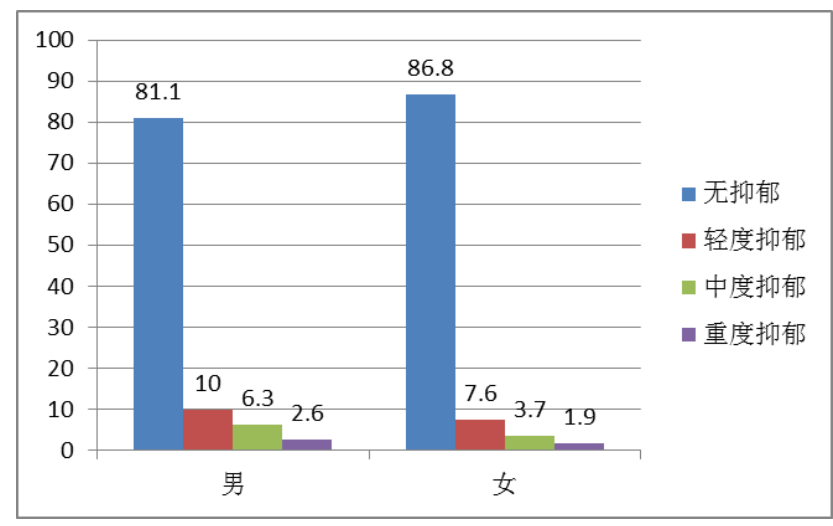

图 1 不同性别抑郁程度分布

表4 不同性别研究生抑郁状况

\begin{tabular}{|c|c|c|c|c|c|c|}
\hline \multicolumn{2}{|c|}{} & 失败感 & 内疚感 & 惩罚感 & 易哭泣 & 总分 \\
\hline \multirow{2}{*}{ 男 } & $\mathrm{M}$ & .37 & .54 & .56 & .44 & 8.22 \\
\cline { 2 - 7 } & $\mathrm{SD}$ & .651 & .549 & .899 & 1.076 & 7.749 \\
\hline \multirow{4}{*}{ 女 } & $\mathrm{M}$ & .22 & .39 & .32 & .24 & 7.08 \\
\cline { 2 - 7 } & $\mathrm{SD}$ & .648 & .560 & .671 & .619 & 7.356 \\
\cline { 2 - 7 } & $\mathrm{Sig}$ & $.012 *$ & $.011 *$ & $.002 * *$ & $.016 *$ & .112 \\
\hline
\end{tabular}

(注: $\mathrm{M}=$ 平均值, $\mathrm{SD}=$ 标准差, $\mathrm{Sig}=$ 显著性水平, 下同)

（2）不同专业研究生抑郁状况

理工科硕士研究生抑郁检出率为 $14.9 \%$, 低于文科生 17\%的抑有检出率。通过 $\mathrm{T}$ 检验发现, 理工科和文科硕士 研究生的抑郁量表得分接近, 不存在显著差异。在各项得 分对比上只有在失败感一项的得分上理工科生显著大于文 科生。

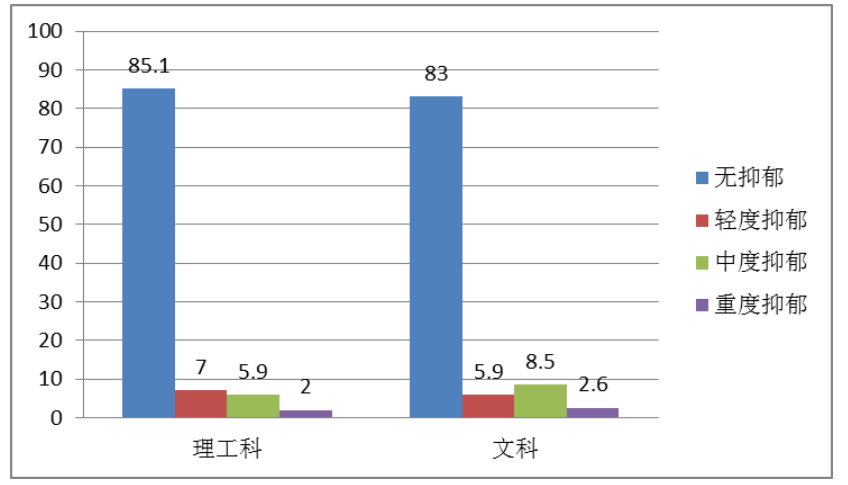

图2 不同专业抑郁程度分布
表5 不同专业研究生抑郁情况

\begin{tabular}{|c|c|c|c|}
\hline \multicolumn{2}{|c|}{} & 失败感 & 总分 \\
\hline \multirow{2}{*}{ 理工 } & $\mathrm{M}$ & .29 & 7.57 \\
\cline { 2 - 4 } & $\mathrm{SD}$ & .598 & 7.422 \\
\hline \multirow{3}{*}{ 文科 } & $\mathrm{M}$ & .13 & 7.51 \\
\cline { 2 - 4 } & $\mathrm{SD}$ & .411 & 7.778 \\
\cline { 2 - 4 } & $\mathrm{Sig}$ & $.004 * *$ & .931 \\
\hline
\end{tabular}

（3）不同年级研究生抑郁状况

据图 3 可知, 研一抑有百分比为 $12.5 \%$, 研二抑郁百 分比为 $22.8 \%$, 研三抑郁百分比为 $10.5 \%$ 。对不同年级各项 目得分进行方差齐性检验。对方差齐性条目采用 LSD 进行 多重比较, 对方差非齐性条目则采用 Tamhane 进行多重比 较分析。表 6 列出不同年级得分存在显著差异的条目。通 过统计分析发现, 研二研三抑郁总分差异非常显著 $(p=0.003)$, 其它比较未发现显著差异。此外, 在惩罚感、 自我嫌弃感、无价值感、食欲改变、疲乏、性欲缺乏这 6 个条目得分上各年级也发现显著差异。研二惩罚感得分最 高, 研三得分最低, 两者差异显著 $(\mathrm{p}=0.030)$ 。自我嫌弃感 得分上研二与研三的差异非常显著 $(\mathrm{p}=0.002)$, 其余相互对 比未发现显著差异。研二无价值感得分最高, 研一最低, 两者差异显著 $(\mathrm{p}=0.019)$, 其余相互对比未发现显著差异。 在食欲改变和疲乏感得分上情况相同, 研二最高, 研一次 之, 研三最低。经两两比较发现, 在这两条目上研二与研 三得分均表现出显著差异 $(\mathrm{p} 1=0.001, \mathrm{p} 2=0.001)$ 。研一与研 二差异不明显, 但与研三差异显著 $(\mathrm{p} 1=0.011, \mathrm{p} 2=0.039)$ 。 最后, 在性欲缺乏这一项上研二得分最高, 与研一差异显 著 $(\mathrm{p}=0.034)$, 与研三差异非常显著 $(\mathrm{p}=0.007)$, 研一研三之 间差异不显著。

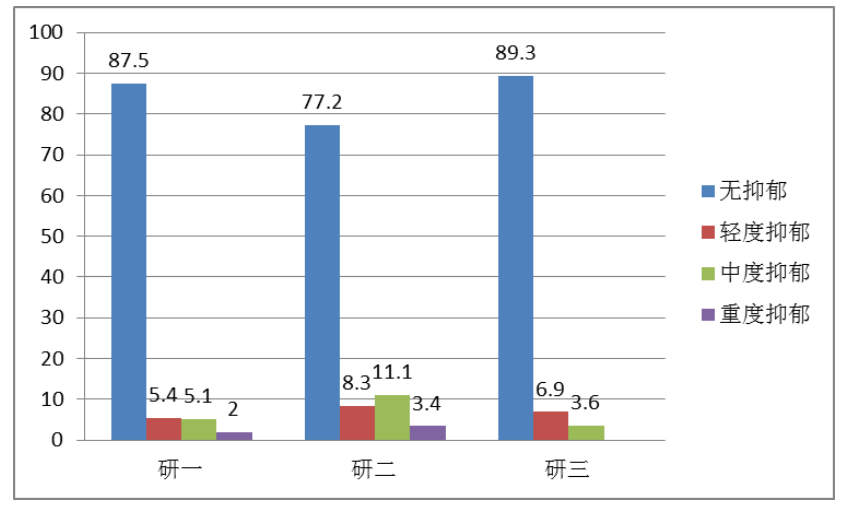

图 3 不同年级抑郁程度分布 
表 6 不同年级研究生抑郁情况

\begin{tabular}{|c|c|c|c|}
\hline 条目 & 研一均值 & 研二均值 & 研三均值 \\
\hline 惩罚感 & .39 & .53 & .25 \\
\hline 自我嫌弃感 & .25 & .43 & .12 \\
\hline 无价值感 & .15 & .32 & .23 \\
\hline 食欲改变 & .31 & .45 & .14 \\
\hline 疲乏 & .51 & .66 & .32 \\
\hline 性欲缺乏 & .15 & .31 & .09 \\
\hline 总分 & 7.16 & 9.01 & 5.55 \\
\hline
\end{tabular}

\section{（4）不同生源研究生抑郁状况}

图 4 显示不同生源研究生的抑郁状况。县镇学生抑郁 百分比为 $18.7 \%$, 农村学生抑郁人数百分比为 $15.4 \%$, 城市 学生抑郁百分比为 $12.2 \%$ 。先对不同生源研究生的数据进 行方差齐性检验, 对方差齐性条目进行方差分析及 LSD 多 重比较, 对方差非齐性条目通过 Tamhane 进行非参数分析。 经数据分析发现在失败感和内疚感上, 不同生源研究生得 分存在显著差异, 其中农村学生失败感得分显著高于城市 学生 $(\mathrm{p}=0.015)$, 县镇学生内疚感得分显著高于城市学生 $(p=0.035)$, 农村学生和县镇学生两者得分差异不显著, 而 在总分上不同生源学生得分未发现显著差异。

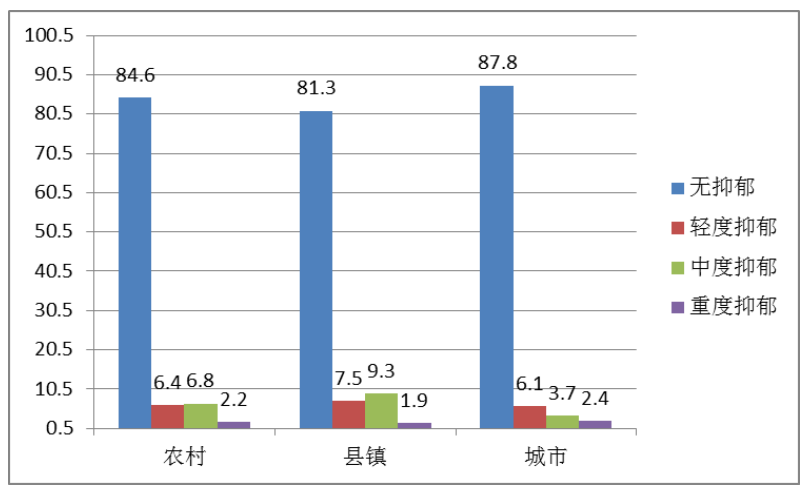

图 4 不同生源抑郁程度分布

表 7 不同生源研究生抑有情况

\begin{tabular}{|c|c|c|c|}
\hline 条目 & 农村均值 & 县镇均值 & 城市均值 \\
\hline 失败感 & .31 & .33 & .15 \\
\hline 内疚感 & .46 & .53 & .32 \\
\hline 总分 & 7.58 & 8.12 & 6.72 \\
\hline
\end{tabular}

\section{3 学生期待获得的帮助}

在运用贝克抑郁量表对学生的抑郁状况进行了解外, 研究者还通过问卷及访谈的形式对硕士研究生在面对抑郁 问题时所期待获得的帮助进行了解。共收集到了 156 条建 议。其中提到希望能得到经济相关方面帮助的有 58 条, 占
总建议的 $38 \%$ 。有 51 条建议提到学校的心理指导不足, 宣 传不够, 希望能多些专业的心理辅导, 举行些有针对性的 心理讲座。有 24 条建议涉及到研究生活动方面的建议, 希 望学校能举办多些学生喜欢的集体活动。其它建议涉及到 就业指导、职业规划、师生关系等。

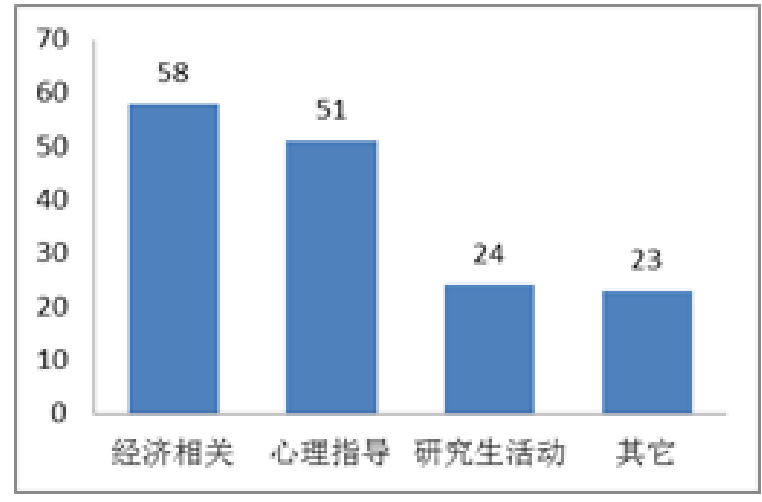

图 5 意见分布

\section{4. 分析讨论}

\section{1 抑郁总体状况}

本次调研抑郁检出率为 $15.6 \%$, 低于 2006 年张月娟[2] 运用流调抑郁自评量表得出的 $21.2 \%$ 抑郁率和 2009 年运用 流调抑郁自评量表在上海市某高校所得出的 $18.6 \%$ 抑郁率 [5], 这表明浙江高校硕士研究生抑郁状况总体上较好。但 $15.6 \%$ 的抑郁检出率说明浙江高校硕士研究生心理健康问 题不容乐观, 相关教育职能部门应给予高度重视。在得分 大于 2 分的条目中, 人数最多的是自责。依据艾里克森人 格发展八阶段理论硕士研究生大都处在 “亲密对孤独的冲 突” 阶段, 在这一阶段学生背负的不仅是自己的梦想, 更 多的还有家庭的未来, 所以当他们认为做得不够好的时候, 往往会失落自责。因此, 在进行硕士研究生心理健康教育 时应基于该阶段的心理发展特点，同时充分考虑性别、年 级和生源等因素, 有区别和有针对性地开展心理健康教育。

\section{2 抑郁状况性别差异}

本次调研中男生抑郁人数比率为 $18.9 \%$, 女生为 $13.2 \%$ 。男生抑郁得分高于女生, 但未发现两者存在显著差 异。2005 年运用贝克抑郁量表对本科生的研究中同样未发 现男女生在抑郁得分上存在显著差异[6]。饶淑璇运用流调 中心抑郁自评量表(CES-D)的测评结果也未发现男女生显 著差异[7]。根据调研数据分析, 男生比女生的抑郁情况严 重, 其主要原因可能在于目前社会给予男生更多的社会责 
任, 男生不得不面对即将到来的家庭压力和工作压力。男 生在努力过程中也更易遭受挫折, 因此男生的失败感、内 疚感和惩罚感均高于女生。另外, 在面对心理问题时女生 会比男生有更积极的心理求助态度。而在传统价值影响下 男性被要求坚强、不轻易表露感情, 这进一步造成男生心 理压力的增大, 使其无法得到有效释放。

\section{3 抑郁状况学科差异}

理工科生抑郁人数百分比为 $14.9 \%$, 文科生百分比为 $17 \%$ 。理工科和文科生的抑郁总分接近, 两者未发现显著 差异。在量表的 21 条条目的对比中, 两者存在显著差异的 条目仅有失败感一条。因此, 本研究认为学科的差异未对 抑有水平造成显著影响。

\section{4 抑郁状况年级差异}

已有研究表明不同年级的研究生心理健康状况存在差 异, 本研究再次证明了这一点。研究发现随着年级的变化 研究生抑郁状况会发生相应改变。在本次调查中研二抑郁 程度最严重, 研三抑郁程度最轻, 三个年级抑郁状况呈倒 $\mathrm{U}$ 型。其原因主要在于: 研一入校时间相对较短, 对研究 生生活充满期待, 课程学习相对轻松, 因而其抑郁得分较 低。研二抑郁程度较高是由于他们面临着论文发表、选题 开题、撰写毕业论文等压力。我们发放该调查问卷的时期 是学年下半学期, 当时研三学生的毕业论文基本已经完成, 很多同学的工作也已落实, 所以他们的抑郁程度相对较低。

\section{5 抑郁状况生源差异}

调研发现, 学生生源差异未显著地影响学生抑郁水平。 在调查样本中来自农村的学生占 $58.5 \%$, 来自县镇的占 $23.5 \%$, 来自城市的占 $18.0 \%$ 。对比其量表得分, 城市学生 得分较低。城市学生抑郁得分较低的原因可能是由于他们 对城市生活更为适应, 较少地感到焦虑。同时很多来自城 市的学生在自己所在城市里求学, 减少了无助感。而来自 县镇和农村的学生面临适应城市生活的问题, 同时他们对 于通过学习改变自身命运抱有更高的期望, 其焦虑感会更 高, 进而导致其失败感和内疚感得分偏高。

\section{6 研究生期待获得的帮助}

从获得的意见分布来看, 对待抑郁这个问题上, 多数 研究生希望能获得经济上的帮助, 这在一定程度上说明经 济压力是导致研究生产生抑郁问题的重要原因。另外, 在 心理指导这一块的意见也占了很大比重, 反应出目前高校 研究生心理援助这一环节的薄弱。从收集的意见上了解到
研究生希望学校能开展各种他们所喜欢的活动, 比如举办 一些团体交友活动。研究生忙于科研, 导致他们与周围人 接触和交流不足, 这样比较容易导致抑有情绪的堆积, 而 开展团体活动有利于这种情绪的释放。其它建议分布较分 散, 涉及到就业指导、职业规划、师生关系等。

\section{5. 应对策略}

\section{1 从身心发展特点出发给予个性化帮助}

本研究发现男生抑郁程度高于女生。一般认为女性对 事物更为敏感, 自尊心更强, 但与男生相比女生也更愿意 向他人倾诉烦恼, 表现出积极的心理求助态度, 针对该特 点要积极引导, 当她们遇到心理困惑或问题时可主动寻求 心理辅导员或导师的帮助。针对男生精力充沛和不善表达 的身心特点高校可多组织诸如足球、篮球、长跑等体育活 动, 通过运动的方式释放他们积蓄在内心的抑郁。针对研 究生间交流不足的问题可以开展一些轻松的团体活动, 在 沟通中疏导压力。同时, 由于研究生不同年级的抑郁状况 存在差异, 需要高校心理健康教育工作有针对性地展开, 针对研一新生首先帮助他们尽快适应新的学习生活, 处理 好新的人际关系, 其次要对其进行心理健康篎查, 发现心 理问题者及时指导和进行后续跟踪; 针对研二较大的学术 压力, 需要导师从学生的兴趣、特长、性格等出发给予个 性化的学术生涯规划, 帮助学生厘清学术研究思路, 制定 可行研究步骤, 为其学术研究指明方向; 针对研三就业或 进一步深造的压力, 要根据学生志趣、能力、家庭等方面 进行职业规划和指导, 帮助研究生树立正确的就业观和升 学观, 尽量减少由于认识不足而造成的心理抑郁。

\section{2 从心理咨询机构着手构建人性化平台}

为提高大学生心理健康水平及生活质量, 高校一般都 设有专门的心理咨询机构并配有专业人员。由于研究生和 本科生在生理和心理上都存在一定差异, 这就需要高校心 理咨询机构设立专门服务研究生的心理咨询部门, 以专业 性来体现研究生心理健康教育的人文性。针对硕士研究生 比较成熟的身心特点, 高校心理咨询机构在研究生心理健 康教育方面可从以下几个方面着手: (1)推进高校心理咨询 机构制度化和规范化建设。努力解决心理咨询机构的人员 数量不足、素质不高、制度不健全等问题, 以满足研究生 的咨询需求。(2)加强高校研究生心理健康教育的宣传。很 多遇到心理问题的研究生不敢去进行心理咨询, 担心泄露 隐私, 被人嘲笑。高校有必要采取更为有效的宣传手段, 让研究生了解心理咨询的职业规范, 消除疑虑, 在面临心 
理问题时能主动寻求帮助。(3)形成人性化的心理咨询机制。 对抑郁症状严重的研究生应持续关注, 并采取心理咨询和 心理治疗等措施, 帮助他们疏导抑郁情绪, 建立心理咨询 师一导师一家长一辅导员一室友 “五位一体” 的机制, 确 保全面、系统、有效的心理援助。

\section{3 从自我认知抓起养成正确人生态度}

自我认知是形成正确人生态度的前提, 个体任何心理 问题的产生、调试和解决都需要通过自我认知来实现。从 个体层面出发, 研究生可从以下几个方面努力: (1)正确认 识自我, 努力完善自我。研究生在融入社会的过程中, 不 少人还在追寻 “我是谁”, “我要成为什么样的人”, “我能 干什么” 等问题的答案。研究生要正确认识自我, 就是要 认清自己的能力, 知道自己的优缺点, 在实践过程中不断 修正理想自我, 完善现实自我, 使双方协调一致。(2)养成 积极的归因方式。个体的归因方式是在后天的学习中逐渐 形成的, 对当时事件的归因直接影响以后对同类事件采取 的应对方式。Needles 指出要重视生活中的正性事件, 因为 个体经历的正性事件越多越容易形成积极的归因方式[8]。 因此, 研究生在进行自我归因训练时要多使自己暴露在正 性事件的环境中。(3)积极学习为人处事之道。师生之间, 朋友之间, 恋人之间, 同事之间的关系处理不好都可能引 发心理问题。研究生相对来说缺少历练, 处事不够成熟, 建议研究生走出去, 以积极的心态面对社会。

\section{4 从人际互动着眼促成和谐师生关系}

和谐师生关系是研究生与导师进行科学研究, 学术探 讨, 学术生涯规划的重要前提, 而师生关系的不融洽则极 易导致研究生心理问题的发生。研究生期间学生和导师的 接触较多, 互动频繁。若有心理问题导师及早发现、介入 可避免许多严重后果的产生, 这也是正确引导、妥善处理 心理危机的重要环节。因此需要, (1)师生共同努力构建和 谐师生关系。首先, 学生要与导师主动交流, 交流内容不 仅可以是学术问题, 也可以是生活情感等方面, 使导师全 面了解自己。其次, 导师要了解关注学生。有时导师在没 有足够了解学生的情况下就给定研究方向, 指定科研任务, 而非学生的专长和兴趣, 那么就可能引起师生之间的隔阂。 再次, 导师还应该从生活上关心学生, 关心学生生活中遇 到的疑惑和困难, 并及时给予指点和帮助。(2)研究生导师 进行相关知识技能培训。学生有心理问题时可能第一个找 的就是导师, 导师的心理指导水平将会对学生产生直接影 响。避免一些导师无法及时发现学生心理问题, 或者发现 问题却不知如何处理的情况, 所以高校需要对导师进行适
当的心理指导培训, 提升导师心理健康指导水平。

\section{5 从经济独立入手减轻学术生活压力}

进一步探究研究生抑郁问题产生的原因, 会发现很多 抑郁情绪直接或间接是由经济原因造成的。研究生作为成 年人不论从心理和生理上都要求独立, 所以很多学生进入 研究生阶段以后已不再向家里要钱, 尝试着部分经济独立 或完全经济独立, 而他们收入来源则主要靠奖助学金、国 家补贴, 导师补贴和兼职获得。面对经济独立, 很多研究 生面临较大压力, 特别是要处理好学业和兼职的问题。高 校可从以下几点出发给予帮助: (1)鼓励研究生学会理财。 常言 “你不理财, 财不理你”, 研究生或多或少会有一些收 入, 如果能较好地规划这部分收入在一定程度上可以减轻 其经济压力。研究生中就不缺乏通过自我理财而实现经济 独立的例子。(2)学校和学院给予经济帮助。高校针对研究 生身心比较成熟的特点, 可以提供助教、兼职思想政治辅 导员、实验室维护员等具有一定技术的岗位, 这样不仅可 以减轻研究生的经济压力, 还可提高其专业岗位能力和社 会适应能力。(3)树立正确的消费观。高校应在研究生群体 中倡导形成勤俭节约的风尚。不铺张浪费, 不过度消费, 践行优秀的民族传统, 树立高尚的人生追求。

\section{参考文献(References)}

[1] C. Holden, "Globalsurvey examines impact of depression," Science, vol. 288, no.5463, pp. 39-40, 2000.

[2] Z. Y. Juan, W. Z. Qi and Z. H. Wei, et al, "Correlation study on depression and negative life events among graduates," Chinese Journal of School Health, vol. 27, no 7, pp.595-596, 2006.

[3] AT. Beck, Steer R Manual for the Beck Depression Inventory, San Antonio, TX: Psychological Corporation, 1996.

[4] W. Zheng, Y. C. Mei and H. Jia, et al, "Reliability and validity of the Chinese version of beck depression inventory-II among depression patients," Chinese Journal of School Health, vol. 25, no.6, pp. 476-479, 2011.

[5] G. Z. Li, "Research on depression of postgraduates in a certain famous university in Shanghai," Chinese Journal of Health Psychology, vol. 17, no. 8, pp. 960-964, 2009.

[6] Y. W. Hui, W. D. Jin and P. Fang, "Application of Chinese vesion of beck depression inventory-II to Chinese first-year college students," Chinese Journal of Clinical Psychology, vol. 20, no. 6, pp.762-764, 2012.

[7] R. S. Xuan, "Research on postgraduate students' depression condition and its adaption strategies," Nanchang University, 2009.

[8] D. J. Needles and L. Y. Abramson, "Positive life events, attributional style, and hopefulness: Testing a model of recovery from depression," Journal of Abnormal Psychology, vol. 2, no.2, pp. 156-165, 1990. 